\title{
Quasilinear Elliptic Equations with Hardy-Sobolev Critical Exponents: Existence and Multiplicity of Nontrivial Solutions
}

\author{
Guanwei Chen \\ School of Mathematics and Statistics, Anyang Normal University, Anyang, Henan 455000, China \\ Correspondence should be addressed to Guanwei Chen; guanweic@163.com
}

Received 30 November 2013; Accepted 12 January 2014; Published 19 February 2014

Academic Editor: Tai-Ping Chang

Copyright ( 2014 Guanwei Chen. This is an open access article distributed under the Creative Commons Attribution License, which permits unrestricted use, distribution, and reproduction in any medium, provided the original work is properly cited.

We study the existence of positive solutions and multiplicity of nontrivial solutions for a class of quasilinear elliptic equations by using variational methods. Our obtained results extend some existing ones.

\section{Introduction and Main Results}

Let us consider the following problem:

$$
\begin{gathered}
-\Delta_{p} u-\mu \frac{|u|^{p^{*}(s)-2}}{|x|^{s}} u=f(x, u), \quad x \in \Omega \backslash\{0\}, \\
u=0, \quad x \in \partial \Omega,
\end{gathered}
$$

where $\Delta_{p} u=\operatorname{div}\left(|\nabla u|^{p-2} \nabla u\right)$ denotes the $p$-Laplacian differential operator, $\Omega$ is an open bounded domain in $\mathbb{R}^{N}(N \geq$ 3) with smooth boundary $\partial \Omega$ and $0 \in \Omega, 0 \leq s<p, p^{*}(s)=$ $p(N-s) /(N-p)$ is the Hardy-Sobolev critical exponent, and $p^{*}=p^{*}(0)=N p /(N-p)$ is the Sobolev critical exponent. Here, we let

$$
\|u\|:=\left(\int_{\Omega}|\nabla u|^{p} d x\right)^{1 / p}
$$

which is equivalent to the usual norm of Sobolev space $W_{0}^{1, p}(\Omega)$ due to the Poincaré inequality. Let

$$
A_{s}(\Omega):=\inf _{u \in W_{0}^{1, p}(\Omega) \backslash\{0\}} \frac{\|u\|^{p}}{\left(\int_{\Omega}\left(|u|^{p^{*}(s)} /|x|^{s}\right) d x\right)^{p / p^{*}(s)}},
$$

which is the best Hardy-Sobolev constant.
In the case where $s=0$ and $\mu=1$ hold, then (1) reduces to the quasilinear elliptic problem:

$$
\begin{gathered}
-\Delta_{p} u-|u|^{p^{*}-2} u=f(x, u), \quad x \in \Omega, \\
u=0, \quad x \in \partial \Omega .
\end{gathered}
$$

Gonçalves and Alves [1] have studied (4) in $\mathbb{R}^{N}$ involving $f(x, u)=h(x) u^{q}, u \geq 0$ and $u \neq \equiv \quad 0$ to obtain existence of positive solutions where $2 \leq p<N, 0<q<p-1$, or $p-1<q<p^{*}-1$ and a suitable $h$. We should mention that problem (4) with $p=2$ has been widely studied since Brézis and Nirenberg; see [2-4] and the references therein.

Ghoussoub and Yuan [5] have studied (1) with $f(x, u)=$ $\lambda|u|^{r-2} u$, where $p<r<p^{*}$. They obtained a positive solution in the case where $\lambda>0, \mu>0$, and $N>\left[p(p-1) r+p^{2}\right] /[p+$ $(p-1)(r-p)]$ (in particular if $N \geq p^{2}$ ) hold. They also obtained a sign-changing solution in the case where $\lambda>0$, $\mu>0$, and $N>[p(p-1) r+p] /[1+(p-1)(r-p)]$ (in particular if $\left.N>p^{3}-p^{2}+p\right)$ hold. For other relevant papers, see $[6-12]$ and the references therein.

We should mention that the energy functional associated with (1) is defined on $W_{0}^{1, p}(\Omega)$, which is not a Hilbert space for $p \neq 2$. Due to the lack of compactness of the embedding in $W_{0}^{1, p}(\Omega) \hookrightarrow L^{p^{*}}(\Omega)$ and $W_{0}^{1, p}(\Omega) \hookrightarrow L^{p^{*}(s)}\left(\Omega,|x|^{-s} d x\right)$, we cannot use the standard variational argument directly. The corresponding energy functional fails to satisfy the classical Palais-Smale ((PS) for short) condition in $W_{0}^{1, p}(\Omega)$. However, 
a local (PS) condition can be established in a suitable range. Then the existence result is obtained via constructing a minimax level within this range and the Mountain Pass Lemma due to Ambrosetti et al. [2] and Rabinowitz [13].

In this paper, we study (1) with a general nonlinearity by using a variational method; besides, we also considerably generalize the results obtained in [5]. In what follows, we always assume that the nonlinearity $f$ satisfies $f(x, 0) \equiv 0$. Let $F(x, t):=\int_{0}^{t} f(x, s) d s, x \in \Omega$. To state our main results, we still need the following assumptions.

$\left(A_{1}\right) f \in C\left(\bar{\Omega} \times \mathbb{R}^{+}, \mathbb{R}\right), \lim _{t \rightarrow 0^{+}}\left(f(x, t) / t^{p-1}\right)=0$, and $\lim _{t \rightarrow \infty}\left(f(x, t) / t^{p^{*}-1}\right)=0$ uniformly for $x \in \bar{\Omega}$.

$\left(A_{2}\right)$ There exists a constant $\rho$ with $\rho>p$ such that

$$
0<\rho F(x, t) \leq f(x, t) t, \quad \forall x \in \bar{\Omega}, \forall t \in \mathbb{R}^{+} \backslash\{0\} .
$$

$\left(A_{3}\right) f \in C(\bar{\Omega} \times \mathbb{R}, \mathbb{R}), \lim _{t \rightarrow 0}\left(f(x, t) /|t|^{p-1}\right)=0$, and $\lim _{|t| \rightarrow \infty}\left(f(x, t) /|t|^{p^{*}-1}\right)=0$ uniformly for $x \in \bar{\Omega}$.

$\left(A_{4}\right)$ There exists a constant $\rho$ with $\rho>p$ such that

$0<\rho F(x, t) \leq f(x, t) t, \quad \forall x \in \bar{\Omega}, \forall t \in \mathbb{R} \backslash\{0\}$.

Now, our main results read as follows.

Theorem 1. Suppose that $N \geq 3,0<\mu<\infty, 0 \leq s<p$, and $1<p<N$ hold. If $\left(A_{1}\right),\left(A_{2}\right)$, and

$$
\rho>\max \left\{p, p^{*}\left(1-\frac{1}{p}\right), p^{*}-\frac{p}{p-1}\right\}
$$

hold, then (1) has at least one positive solution.

Theorem 2. Suppose that $N \geq 3,0<\mu<\infty, 0 \leq s<p$, and $1<p<N$ hold. If $\left(A_{3}\right),\left(A_{4}\right)$, and (7) hold, then (1) has at least two distinct nontrivial solutions.

Noting that $\rho>p$ and $p^{2} \leq N$ imply that (7) holds, therefore, we have the following corollaries.

Corollary 3. Suppose that $0<\mu<\infty, 0 \leq s<p, p^{2} \leq N$, and $1<p<N$. Moreover, $\left(A_{1}\right)$ and $\left(A_{2}\right)$ hold; then $(1)$ has at least one positive solution.

Corollary 4. Suppose that $0<\mu<\infty, 0 \leq s<p, p^{2} \leq N$, and $1<p<N$. Moreover, $\left(A_{3}\right)$ and $\left(A_{4}\right)$ hold; then $(1)$ has at least two distinct nontrivial solutions.

Remark 5. Theorem 1 generalizes Theorem 1.3 in [5], where the author only studied the special situation that $f(x, u)=$ $\lambda|u|^{r-2} u, p<r<p^{*}$. There are functions $f$ satisfying the assumptions of our Theorem 1 and not satisfying those in [5]. Let

$$
f(x, t):=g(x)|t|^{k-2} t+a|t|^{l-2} t, \quad(x, t) \in \bar{\Omega} \times \mathbb{R},
$$

where $g(x)>0, g \in L^{\infty}(\Omega), a>0$, and $p<k<l<p^{*}$. Obviously, $f$ satisfies all the conditions of Theorem 1 in this paper, while it does not satisfy the conditions of Theorem 1.3 in [5].
The rest of this paper is organized as follows. In Section 2, we give some preliminary lemmas, which are useful in the proofs of our main results. In Section 3, we give the detailed proofs of our main results.

\section{Preliminaries}

In what follows, we let $\|\cdot\|_{p}$ denote the norm in $L^{p}(\Omega)$. It is obvious that the values of $f(x, t)$ for $t<0$ are irrelevant in Theorem 1, so we may define

$$
f(x, t) \equiv 0 \quad \text { for } x \in \Omega, t \leq 0 .
$$

We firstly consider the existence of nontrivial solutions to the problem:

$$
\begin{gathered}
-\Delta_{p} u=\mu \frac{\left(u^{+}\right)^{p^{*}(s)-1}}{|x|^{s}}+f(x, u), \quad x \in \Omega \backslash\{0\}, \\
u=0, \quad x \in \partial \Omega .
\end{gathered}
$$

The energy functional corresponding to (10) is given by

$$
\begin{aligned}
I(u)= & \frac{1}{p} \int_{\Omega}|\nabla u|^{p} d x-\frac{\mu}{p^{*}(s)} \int_{\Omega} \frac{\left(u^{+}\right)^{p^{*}(s)}}{|x|^{s}} d x \\
& -\int_{\Omega} F(x, u) d x, \quad u \in W_{0}^{1, p}(\Omega) .
\end{aligned}
$$

By Hardy-Sobolev inequalities (see $[5,14])$ and $\left(A_{1}\right)$, we know $I \in C^{1}\left(W_{0}^{1, p}(\Omega), \mathbb{R}\right)$. Now it is well known that there exists a one-to-one correspondence between the weak solutions of (10) and the critical points of $I$ on $W_{0}^{1, p}(\Omega)$. More precisely we say that $u \in W_{0}^{1, p}(\Omega)$ is a weak solution of (10), if, for any $v \in W_{0}^{1, p}(\Omega)$, there holds

$$
\begin{aligned}
\left\langle I^{\prime}(u), v\right\rangle= & \int_{\Omega}|\nabla u|^{p-2}(\nabla u, \nabla v) d x-\mu \int_{\Omega} \frac{\left(u^{+}\right)^{p^{*}(s)-1}}{|x|^{s}} v d x \\
& -\int_{\Omega} f(x, u) v d x=0 .
\end{aligned}
$$

Lemma 6 (see [15]). If $f_{n} \rightarrow f$ a.e. in $\Omega$ and $\left\|f_{n}\right\|_{p} \leq C<\infty$ for all $n$ and some $0<p<\infty$, then

$$
\lim _{n \rightarrow \infty}\left(\left\|f_{n}\right\|_{p}^{p}-\left\|f_{n}-f\right\|_{p}^{p}\right)=\|f\|_{p}^{p}
$$

Lemma 7. For any $a>0,0 \leq b \leq 1$, and $\lambda \geq 1$, we have $(a+b)^{\lambda} \leq a^{\lambda}+\lambda(a+1)^{\lambda-1} b$.

Proof. Let

$$
h(x):=(a+x)^{\lambda}-a^{\lambda}-\lambda(a+1)^{\lambda-1} x .
$$

Clearly, $h^{\prime}(x)<0, x \in(0,1)$, so $h(b) \leq h(0)=0$. 
Lemma 8 (see [5]). If $0 \leq s<p$ hold, then we have

(i) $A_{s}(\Omega)$ is independent of $\Omega$, and will henceforth be denoted by $A_{s}$;

(ii) $A_{s}(\Omega)$ is attained when $\Omega=\mathbb{R}^{N}$ by the functions

$$
\begin{aligned}
l_{\varepsilon}(x)= & \left(\varepsilon(N-s)\left(\frac{N-p}{p-1}\right)^{p-1}\right)^{(N-p) / p(p-s)} \\
& \times\left(\varepsilon+|x|^{(p-s) /(p-1)}\right)^{(p-N) /(p-s)}
\end{aligned}
$$

for some $\varepsilon>0$. Moreover, the functions $l_{\varepsilon}(x)$ are the only positive radial solutions of

$$
-\Delta_{p} u=\frac{u^{p^{*}(s)-1}}{|x|^{s}}
$$

in $\mathbb{R}^{N}$, and satisfy

$$
\int_{\mathbb{R}^{N}}\left|\nabla l_{\varepsilon}\right|^{p} d x=\int_{\mathbb{R}^{N}} \frac{\left|l_{\varepsilon}\right|^{p^{*}(s)}}{|x|^{s}} d x=\left(A_{s}(\Omega)\right)^{(N-s) /(p-s)} .
$$

Lemma 9. If $\left(A_{1}\right),\left(A_{2}\right)$, and $0<c<((p-s) / p(N-$ s)) $A_{s}^{(N-s) /(p-s)} \mu^{(p-N) /(p-s)}$ hold, then I satisfies $(P S)_{c}$ condition.

Proof. Suppose that $\left\{u_{n}\right\}$ is a (PS) sequence in $W_{0}^{1, p}(\Omega)$. By $\left(A_{2}\right)$, we have

$$
\begin{aligned}
c+ & 1+o(1)\left\|u_{n}\right\| \\
\geq & I\left(u_{n}\right)-\frac{1}{\theta}\left\langle I^{\prime}\left(u_{n}\right), u_{n}\right\rangle \\
= & \left(\frac{1}{p}-\frac{1}{\theta}\right)\left\|u_{n}\right\|^{p}+\left(\frac{1}{\theta}-\frac{1}{p^{*}(s)}\right) \mu \int_{\Omega} \frac{\left(u_{n}^{+}\right)^{p^{*}(s)}}{|x|^{s}} d x \\
& -\int_{\Omega}\left(F\left(x, u_{n}\right)-\frac{1}{\theta} f\left(x, u_{n}\right) u_{n}\right) d x \\
\geq & \left(\frac{1}{p}-\frac{1}{\theta}\right)\left\|u_{n}\right\|^{p},
\end{aligned}
$$

where $\theta=\min \left\{\rho, p^{*}(s)\right\}$. Hence we conclude that $\left\{u_{n}\right\}$ is a bounded sequence in $W_{0}^{1, p}(\Omega)$. So there exists $u \in W_{0}^{1, p}(\Omega)$; going if necessary to a subsequence, we have

$$
u_{n} \rightarrow u \quad \text { in } W_{0}^{1, p}(\Omega),
$$

$$
\begin{array}{r}
u_{n} \longrightarrow u \text { in } L^{\gamma}(\Omega) \quad\left(1<\gamma<p^{*}\right), \\
u_{n} \longrightarrow u \quad \text { a.e. in } \Omega, \\
n \longrightarrow \infty .
\end{array}
$$

By the continuity of embedding, we have $\left\|u_{n}\right\|_{p^{*}}^{p^{*}} \leq C_{1}<\infty$. From [5], going if necessary to a subsequence, one can get that

$$
\begin{gathered}
\nabla u_{n} \longrightarrow \nabla u \quad \text { a.e. in } \Omega, \\
\frac{u_{n}}{x} \longrightarrow \frac{u}{x} \quad \text { weakly in } L^{p}(\Omega), \\
\int_{\Omega} \frac{\left|u_{n}\right|^{p^{*}(s)-2} u_{n}}{|x|^{s}} v d x \rightarrow \int_{\Omega} \frac{|u|^{p^{*}(s)-2} u}{|x|^{s}} v d x, \quad \forall v \in W_{0}^{1, p}(\Omega)
\end{gathered}
$$

as $n \rightarrow \infty$. By $\left(A_{1}\right)$, we know that for any $\varepsilon>0$ there exists $a(\varepsilon)>0$ such that

$$
|f(x, t) t| \leq a(\varepsilon)+\frac{1}{2 C_{1}} \varepsilon|t|^{p^{*}} \quad \text { for }(x, t) \in \bar{\Omega} \times(0,+\infty) .
$$

Set $\delta:=\varepsilon / 2 a(\varepsilon)>0$. When $E \subset \Omega$, meas $(E)<\delta$, we get

$$
\begin{aligned}
\left|\int_{E} f\left(x, u_{n}\right) u_{n} d x\right| & \leq \int_{E} a(\varepsilon) d x+\frac{\varepsilon}{2 C_{1}} \int_{E}\left|u_{n}\right|^{p^{*}} d x \\
& <\varepsilon \longrightarrow 0 \text { as meas }(E) \longrightarrow 0
\end{aligned}
$$

It follows from Vitali's theorem that

$$
\int_{\Omega} f\left(x, u_{n}\right) u_{n} d x \rightarrow \int_{\Omega} f(x, u) u d x \quad \text { as } n \longrightarrow \infty \text {. }
$$

Similarly, we can also get

$$
\int_{\Omega} F\left(x, u_{n}\right) d x \longrightarrow \int_{\Omega} F(x, u) d x \quad \text { as } n \longrightarrow \infty .
$$

Since $I^{\prime}\left(u_{n}\right) \rightarrow 0$, we have

$$
\begin{aligned}
& \left\langle I^{\prime}\left(u_{n}\right), u_{n}\right\rangle \\
& \quad=\left\|u_{n}\right\|^{p}-\mu \int_{\Omega} \frac{\left(u_{n}^{+}\right)^{p^{*}(s)}}{|x|^{s}} d x-\int_{\Omega} f\left(x, u_{n}\right) u_{n} d x=o(1) .
\end{aligned}
$$

Let $v_{n}:=u_{n}-u$, which together with Lemma 6 implies

$$
\begin{aligned}
\left\|v_{n}\right\|^{p} & +\|u\|^{p}-\mu \int_{\Omega} \frac{\left(v_{n}^{+}\right)^{p^{*}(s)}}{|x|^{s}} d x-\mu \int_{\Omega} \frac{\left(u^{+}\right)^{p^{*}(s)}}{|x|^{s}} d x \\
& -\int_{\Omega} f(x, u) u d x=o(1) .
\end{aligned}
$$

From (20), we can obtain

$$
\begin{aligned}
\lim _{n \rightarrow \infty}\left\langle I^{\prime}\left(u_{n}\right), u\right\rangle= & \|u\|^{p}-\mu \int_{\Omega} \frac{\left(u^{+}\right)^{p^{*}(s)}}{|x|^{s}} d x \\
& -\int_{\Omega} f(x, u) u d x=0 .
\end{aligned}
$$


Note that $I\left(u_{n}\right) \rightarrow c$ as $n \rightarrow \infty$, which together with Lemma 6 implies

$$
\begin{aligned}
I\left(u_{n}\right)= & \frac{1}{p}\left\|v_{n}\right\|^{p}+\frac{1}{p}\|u\|^{p}-\frac{\mu}{p^{*}(s)} \int_{\Omega} \frac{\left(v_{n}^{+}\right)^{p^{*}(s)}}{|x|^{s}} d x \\
& -\frac{\mu}{p^{*}(s)} \int_{\Omega} \frac{\left(u^{+}\right)^{p^{*}(s)}}{|x|^{s}} d x-\int_{\Omega} F(x, u) d x+o(1) \\
= & I(u)+\frac{1}{p}\left\|v_{n}\right\|^{p}-\frac{\mu}{p^{*}(s)} \int_{\Omega} \frac{\left(v_{n}^{+}\right)^{p^{*}(s)}}{|x|^{s}} d x+o(1) \\
= & c+o(1) .
\end{aligned}
$$

Therefore, one gets that

$$
I(u)+\frac{1}{p}\left\|v_{n}\right\|^{p}-\frac{\mu}{p^{*}(s)} \int_{\Omega} \frac{\left(v_{n}^{+}\right)^{p^{*}(s)}}{|x|^{s}} d x=c+o(1) .
$$

From (26) and (27), we have

$$
\left\|v_{n}\right\|^{p}-\mu \int_{\Omega} \frac{\left(v_{n}^{+}\right)^{p^{*}(s)}}{|x|^{s}} d x=o(1) ;
$$

then $\left\|v_{n}\right\|^{p} \rightarrow 0$ as $n \rightarrow \infty$. Otherwise, there exists a subsequence (still denoted by $v_{n}$ ) such that

$$
\lim _{n \rightarrow \infty}\left\|v_{n}\right\|^{p}=k, \quad \lim _{n \rightarrow \infty} \mu \int_{\Omega} \frac{\left(v_{n}^{+}\right)^{p^{*}(s)}}{|x|^{s}} d x=k, \quad k>0 .
$$

By (3), we have

$$
\left\|v_{n}\right\|^{p} \geq A_{s}\left(\int_{\Omega} \frac{\left(v_{n}^{+}\right)^{p^{*}(s)}}{|x|^{s}} d x\right)^{p / p^{*}(s)}, \quad \forall n \in \mathbb{N} ;
$$

then $k \geq A_{s}(k / \mu)^{p / p^{*}(s)}$. That is, $k \geq A_{s}^{(N-s) /(p-s)} \mu^{(p-N) /(p-s)}$. It follows from (29) and $c<((p-s) / p(N-$ $s)) A_{s}^{(N-s) /(p-s)} \mu^{(p-N) /(p-s)}$ that $I(u)<0$. However, we have $I(u) \geq 0$ by $(27)$ and $\left(A_{2}\right)$. We get a contradiction. Therefore, we can obtain

$$
\left\|v_{n}\right\|^{p} \longrightarrow 0 \quad \text { as } n \longrightarrow \infty .
$$

From the discussion above, $I$ satisfies $(\mathrm{PS})_{c}$ condition.

In the following, we shall give some estimates for the extremal functions. Let

$$
\begin{gathered}
C_{\varepsilon}:=\left[\varepsilon(N-s)\left(\frac{N-p}{p-1}\right)^{p-1}\right]^{(N-p) / p(p-s)}, \\
U_{\varepsilon}(x):=\frac{l_{\varepsilon}(x)}{C_{\varepsilon}} .
\end{gathered}
$$

Define a function $\varphi \in C_{0}^{\infty}(\Omega)$ such that $\varphi(x)=1$ for $|x| \leq R$, $\varphi(x)=0$ for $|x| \geq 2 R, 0 \leq \varphi(x) \leq 1$, where $B_{2 R}(0) \subset \Omega$. Set

$$
\begin{gathered}
u_{\varepsilon}(x)=\varphi(x) U_{\varepsilon}(x), \\
v_{\varepsilon}(x)=\frac{u_{\varepsilon}(x)}{\left(\int_{\Omega}\left(\left|u_{\varepsilon}\right|^{p^{*}(s)} /|x|^{s}\right)\right)^{1 / p^{*}(s)}},
\end{gathered}
$$

so that $\int_{\Omega}\left(\left|v_{\varepsilon}\right|^{p^{*}(s)} /|x|^{s}\right)=1$. Then, by using the argument as used in [5], we can get the following results:

$$
\begin{gathered}
A_{s}+C_{2} \varepsilon^{(N-p) /(p-s)} \leq \|\left. v_{\varepsilon}\right|^{p} \leq A_{s}+C_{3} \varepsilon^{(N-p) /(p-s)}, \\
C_{4} \varepsilon^{(N-p) r / p(p-s)} \leq \int_{\Omega}\left|v_{\varepsilon}\right|^{r} d x \leq C_{5} \varepsilon^{(N-p) r / p(p-s)}, \\
1 \leq r<p^{*}\left(1-\frac{1}{p}\right), \\
C_{4} \varepsilon^{(N-p) r / p(p-s)}|\ln \varepsilon| \leq \int_{\Omega}\left|v_{\varepsilon}\right|^{r} d x \leq C_{5} \varepsilon^{(N-p) r / p(p-s)}|\ln \varepsilon|, \\
r=p^{*}\left(1-\frac{1}{p}\right), \\
C_{4} \varepsilon^{((p-1) /(p-s))(N-r(N-p) / p)} \quad \\
\leq \int_{\Omega}\left|v_{\varepsilon}\right|^{r} d x \leq C_{5} \varepsilon^{((p-1) /(p-s))(N-r(N-p) / p),} \\
p^{*}\left(1-\frac{1}{p}\right)<r<p^{*} .
\end{gathered}
$$

Moreover, by using the Sobolev embedding theorem and (36), one can deduce

$$
\int_{\Omega}\left|v_{\varepsilon}\right|^{p^{*}} d x \leq C_{6} A_{s}^{N /(N-p)} \text { for } \varepsilon \longrightarrow 0^{+} .
$$

Lemma 10. Suppose that $0 \leq s<p$. If $\left(A_{1}\right),\left(A_{2}\right)$ and (7) hold, then there exists $u_{0} \in W_{0}^{1, p}(\Omega), u_{0} \neq \equiv$, such that

$$
\sup _{t \geq 0} I\left(t u_{0}\right)<\frac{p-s}{p(N-s)} A_{s}^{(N-s) /(p-s)} \mu^{(p-N) /(p-s)} .
$$

Proof. We consider the functions

$$
\begin{gathered}
g(t)=I\left(t v_{\varepsilon}\right)=\frac{t^{p}}{p}\left\|v_{\varepsilon}\right\|^{p}-\mu \frac{t^{p^{*}(s)}}{p^{*}(s)}-\int_{\Omega} F\left(x, t v_{\varepsilon}\right) d x, \\
\bar{g}(t)=\frac{t^{p}}{p}\left\|v_{\varepsilon}\right\|^{p}-\mu \frac{t^{p^{*}(s)}}{p^{*}(s)} .
\end{gathered}
$$

Since $\lim _{t \rightarrow \infty} g(t)=-\infty, g(0)=0$, and $g(t)>0$ for $t$ small enough, $\sup _{t \geq 0} g(t)$ is attained for some $t_{\varepsilon}>0$. Therefore, we have

$$
\begin{aligned}
0 & =g^{\prime}\left(t_{\varepsilon}\right) \\
& =t_{\varepsilon}^{p-1}\left(\left\|v_{\varepsilon}\right\|^{p}-\mu t_{\varepsilon}^{p^{*}(s)-p}-\frac{1}{t_{\varepsilon}^{p-1}} \int_{\Omega} f\left(x, t_{\varepsilon} v_{\varepsilon}\right) v_{\varepsilon} d x\right),
\end{aligned}
$$

and hence

$$
\left\|v_{\varepsilon}\right\|^{p}=\mu t_{\varepsilon}^{p^{*}(s)-p}+\frac{1}{t_{\varepsilon}^{p-1}} \int_{\Omega} f\left(x, t_{\varepsilon} v_{\varepsilon}\right) v_{\varepsilon} d x \geq \mu t_{\varepsilon}^{p^{*}(s)-p} .
$$


Therefore, we obtain

$$
t_{\varepsilon} \leq\left(\frac{\left\|v_{\varepsilon}\right\|^{p}}{\mu}\right)^{1 /\left(p^{*}(s)-p\right)}:=t_{\varepsilon}^{0} .
$$

By $\left(A_{1}\right)$, we can easily get

$$
|f(x, t)| \leq \varepsilon t^{p^{*}-1}+d(\varepsilon) t^{p-1}, \quad d(\varepsilon)>0 .
$$

Hence, we can get

$$
\left\|v_{\varepsilon}\right\|^{p} \leq \mu t_{\varepsilon}^{p^{*}(s)-p}+\varepsilon \int_{\Omega}\left|t_{\varepsilon}\right|^{p^{*}-p}\left|v_{\varepsilon}\right|^{p^{*}} d x+d(\varepsilon) \int_{\Omega}\left|v_{\varepsilon}\right|^{p} d x .
$$

By (36)-(38), when $\varepsilon$ is small enough, we conclude that

$$
t_{\varepsilon}^{p^{*}(s)-p} \geq \frac{A_{s}}{2} .
$$

On the one hand, from Lemma 7 and (36), it follows that

$$
\left\|v_{\varepsilon}\right\|^{p(N-s) /(p-s)} \leq A_{s}^{(N-s) /(p-s)}+C_{7} \varepsilon^{(N-s) /(p-s)} .
$$

On the other hand, the function $\bar{g}(t)$ attains its maximum at $t_{\varepsilon}^{0}$ and is increasing in the interval $\left[0, t_{\varepsilon}^{0}\right]$. Note that $\left(A_{2}\right)$ implies $F(x, t) \geq C_{8}|t|^{\rho}$, which together with (36), (46), and (47) implies that

$$
\begin{aligned}
g\left(t_{\varepsilon}\right) \leq & \bar{g}\left(t_{\varepsilon}^{0}\right)-\int_{\Omega} F\left(x, t_{\varepsilon} v_{\varepsilon}\right) d x \\
= & \frac{p-s}{p(N-s)}\left\|v_{\varepsilon}\right\|^{p(N-s) /(p-s)} \mu^{(p-N) /(p-s)} \\
& -\int_{\Omega} F\left(x, t_{\varepsilon} v_{\varepsilon}\right) d x \\
\leq & \frac{p-s}{p(N-s)} A_{s}^{(N-s) /(p-s)} \mu^{(p-N) /(p-s)} \\
& +C_{9} \mu^{(p-N) /(p-s)} \varepsilon^{(N-p) /(p-s)} \\
& -C_{8}\left(\frac{A_{s}}{2}\right)^{\rho /\left(p^{*}(s)-p\right)} \int_{\Omega}\left|v_{\varepsilon}\right|^{\rho} d x .
\end{aligned}
$$

Furthermore, from (7) and (37), we get

$$
\int_{\Omega}\left|v_{\varepsilon}\right|^{\rho} d x \geq C_{4} \varepsilon^{((p-1) /(p-s))(N-\rho(N-p) / p)} .
$$

By (7), we have $\rho>p^{*}-p /(p-1)$, which implies

$$
\frac{N-p}{p-s}>\frac{p-1}{p-s}\left(N-\frac{\rho(N-p)}{p}\right) \text {. }
$$

Therefore, by choosing $\varepsilon$ small enough, we have

$$
\sup _{t \geq 0} I\left(t v_{\varepsilon}\right)=g\left(t_{\varepsilon}\right)<\frac{p-s}{p(N-s)} A_{s}^{(N-s) /(p-s)} \mu^{(p-N) /(p-s)} .
$$

Hence, the proof of the lemma is completed by taking $u_{0}=$ $v_{\varepsilon}$.

\section{Proofs of Main Results}

Proof of Theorem 1. Let $X:=W_{0}^{1, p}(\Omega)$. From the Sobolev and Hardy-Sobolev inequalities, we can easily get

$$
\begin{array}{r}
\|u\|_{p}^{p} \leq C\|u\|^{p}, \quad \int_{\Omega} \frac{|u|^{p^{*}(s)}}{|x|^{s}} d x \leq C\|u\|^{p^{*}(s)}, \\
\|u\|_{p^{*}}^{p^{*}} \leq C\|u\|^{p^{*}}, \\
\forall u \in X .
\end{array}
$$

It follows from $\left(A_{1}\right)$ that

$$
\begin{array}{r}
\exists \delta_{1}>0, \quad \text { s.t. }|f(t)|<t^{p^{*}-1}, \quad \forall t>\delta_{1} ; \\
\forall \varepsilon>0, \quad \exists 0<\delta_{2}<\delta_{1}, \quad \text { s.t. }|f(t)|<\varepsilon t^{p-1}, \\
\forall t \in\left(0, \delta_{2}\right) \\
\exists M>0, \quad \text { s.t. }|f(t)| \leq M, \quad \forall t \in\left[\delta_{2}, \delta_{1}\right]
\end{array}
$$

uniformly for all $x \in \bar{\Omega}$. Therefore, we deduce that

$$
|f(x, t)| \leq t^{p^{*}-1}+\varepsilon t^{p^{-1}}+M \leq \varepsilon t^{p-1}+\left(1+M \delta_{2}^{1-p^{*}}\right) t^{p^{*}-1}
$$

for all $t \in \mathbb{R}^{+}$and for $x \in \bar{\Omega}$. Then one gets

$$
|F(x, t)| \leq \frac{1}{p} \varepsilon|t|^{p}+C_{10}|t|^{p^{*}}
$$

for all $t \in \mathbb{R}$ and for $x \in \bar{\Omega}$. By (52) and (55) we have

$$
\begin{aligned}
I(u) & =\frac{1}{p}\|u\|^{p}-\frac{\mu}{p^{*}(s)} \int_{\Omega} \frac{\left(u^{+}\right)^{p^{*}(s)}}{|x|^{s}} d x-\int_{\Omega} F(x, u) d x \\
& \geq \frac{1}{p}\|u\|^{p}-\frac{C \mu}{p^{*}(s)}\left\|u^{+}\right\|^{p^{*}(s)}-\frac{C}{p} \varepsilon\|u\|^{p}-C C_{10}\|u\|^{p^{*}}
\end{aligned}
$$

for $\varepsilon$ small enough. So there exists $\beta>0$ such that

$$
\begin{array}{r}
I(u) \geq \beta \quad \forall u \in \partial B_{r}=\left\{u \in W_{0}^{1, p}(\Omega),\|u\|=r\right\}, \\
r>0 \text { small enough. }
\end{array}
$$

By Lemma 10 , there exists $u_{0} \in W_{0}^{1, p}(\Omega)$ with $u_{0} \neq \equiv 0$ such that

$$
\sup _{t \geq 0} I\left(t u_{0}\right)<\frac{p-s}{p(N-s)} A_{s}^{(N-s) /(p-s)} \mu^{(p-N) /(p-s)} .
$$

It follows from the nonnegativity of $F(x, t)$ that

$$
\begin{aligned}
I\left(t u_{0}\right)= & \frac{1}{p} t^{p}\left\|u_{0}\right\|^{p}-\frac{\mu}{p^{*}(s)} t^{p^{*}(s)} \int_{\Omega} \frac{\left(u_{0}^{+}\right)^{p^{*}(s)}}{|x|^{s}} d x \\
& -\int_{\Omega} F\left(x, t u_{0}\right) d x \\
\leq & \frac{1}{p} t^{p}\left\|u_{0}\right\|^{p}-\frac{\mu}{p^{*}(s)} t^{p^{*}(s)} \int_{\Omega} \frac{\left(u_{0}^{+}\right)^{p^{*}(s)}}{|x|^{s}} d x .
\end{aligned}
$$


Therefore, $\lim _{t \rightarrow+\infty} I\left(t u_{0}\right) \rightarrow-\infty$, so we can choose $t_{0}>0$ such that

$$
\left\|t_{0} u_{0}\right\|>r, \quad I\left(t_{0} u_{0}\right) \leq 0
$$

By virtue of the Mountain Pass Lemma in [16], there is a sequence $\left\{u_{n}\right\} \subset X$ satisfying

$$
I\left(u_{n}\right) \longrightarrow c \geq \beta, \quad I^{\prime}\left(u_{n}\right) \longrightarrow 0,
$$

where

$$
\begin{gathered}
c=\inf _{h \in \Gamma} \max _{t \in[0,1]} I(h(t)), \\
\Gamma=\left\{h \in C([0,1], X) \mid h(0)=0, h(1)=t_{0} u_{0}\right\} .
\end{gathered}
$$

Note that

$$
\begin{aligned}
0<\beta \leq c & =\inf _{h \in \Gamma} \max _{t \in[0,1]} I(h(t)) \leq \max _{t \in[0,1]} I\left(t t_{0} u_{0}\right) \leq \sup _{t \geq 0} I\left(t u_{0}\right) \\
& <\frac{p-s}{p(N-s)} A_{s}^{(N-s) /(p-s)} \mu^{(p-N) /(p-s)} .
\end{aligned}
$$

By Lemma 9 we can assume that $u_{n} \rightarrow u$ in $W_{0}^{1, p}(\Omega)$. From the continuity of $I^{\prime}$, we know that $u$ is a weak solution of problem (10). Then $\left\langle I^{\prime}(u), u^{-}\right\rangle=0$, where $u^{-}=\min \{u, 0\}$. Thus $u \geq 0$. Therefore, $u$ is a nonnegative solution of (1). By the Strong Maximum Principle [17], $u$ is a positive solution of problem (1). Therefore, Theorem 1 holds.

Proof of Theorem 2. By Theorem 1, we know that (1) has a positive solution $u_{1}$. Set

$$
g(x, t):=-f(x,-t) \quad \text { for } t \in \mathbb{R} .
$$

It follows from Theorem 1 that the equation

$$
-\Delta_{p} u=\mu \frac{\left|u^{+}\right|^{p^{*}(s)-2}}{|x|^{s}} u+g(x, u)
$$

has at least one positive solution $v$. Let $u_{2}=-v$; then $u_{2}$ is a solution of

$$
-\Delta_{p} u=\mu \frac{\left|u^{+}\right|^{p^{*}(s)-2}}{|x|^{s}} u+f(x, u) .
$$

It is obvious that $u_{1} \neq 0, u_{2} \neq 0$, and $u_{1} \neq u_{2}$. So (1) has at least two nontrivial solutions. Therefore, Theorem 2 holds.

\section{Conflict of Interests}

The author declares that there is no conflict of interests regarding the publication of this paper.

\section{Acknowledgments}

The author thanks the referees and the editors for their helpful comments and suggestions. Research is supported by the Tianyuan Fund for Mathematics of NSFC (Grant no. 11326113) and the Key Project of Natural Science Foundation of Educational Committee of Henan Province of China (Grant no. 13A110015).

\section{References}

[1] J. V. Gonçalves and C. O. Alves, "Existence of positive solutions for $m$-Laplacian equations in $\mathbb{R}^{N}$ involving critical Sobolev exponents," Nonlinear Analysis: Theory, Methods \& Applications, vol. 32, no. 1, pp. 53-70, 1998.

[2] A. Ambrosetti, H. Brezis, and G. Cerami, "Combined effects of concave and convex nonlinearities in some elliptic problems," Journal of Functional Analysis, vol. 122, no. 2, pp. 519-543, 1994.

[3] H. Brézis and L. Nirenberg, "Positive solutions of nonlinear elliptic equations involving critical Sobolev exponents," Communications on Pure and Applied Mathematics, vol. 36, no. 4, pp. 437-477, 1983.

[4] E. Jannelli, "The role played by space dimension in elliptic critical problems," Journal of Differential Equations, vol. 156, no. 2, pp. 407-426, 1999.

[5] N. Ghoussoub and C. Yuan, "Multiple solutions for quasi-linear PDEs involving the critical Sobolev and Hardy exponents," Transactions of the American Mathematical Society, vol. 352, no. 12, pp. 5703-5743, 2000.

[6] G. Chen and S. Ma, "On the quasilinear elliptic problem with a Hardy-Sobolev critical exponent," Dynamics of Partial Differential Equations, vol. 8, no. 3, pp. 225-237, 2011.

[7] G. Chen and S. Ma, "Existence and multiplicity of solutions for quasilinear elliptic equations," preprinted.

[8] G. Chen and S. Ma, "Multiple positive solutions for a quasilinearelliptic equation involving Hardy term and Hardy-Sobolev critical exponent," preprinted.

[9] Y. Deng and L. Jin, "Multiple positive solutions for a quasilinear nonhomogeneous Neumann problems with critical Hardy exponents," Nonlinear Analysis: Theory, Methods \& Applications, vol. 67, no. 12, pp. 3261-3275, 2007.

[10] H. Egnell, "Positive solutions of semilinear equations in cones," Transactions of the American Mathematical Society, vol. 330, no. 1, pp. 191-201, 1992.

[11] N. Ghoussoub and X. S. Kang, "Hardy-Sobolev critical elliptic equations with boundary singularities," Annales de l'Institut Henri Poincaré, vol. 21, no. 6, pp. 767-793, 2004.

[12] E. A. B. Silva, J. C. N. Padua, and S. H. M. Soares, "Positive solutions of critical semilinear problems involving a sublinear term at the origin," Cadernos de Mathematica, vol. 5, pp. 245262, 2004.

[13] P. H. Rabinowitz, Minimax Methods in Critical Point Theory with Applications to Differential Equations, vol. 65 of CBMS Regional Conference Series in Mathematics, American Mathematical Society, Washington, DC, USA, 1986.

[14] L. Caffarelli, R. Kohn, and L. Nirenberg, "First order interpolation inequalities with weights," Compositio Mathematica, vol. 53, no. 3, pp. 259-275, 1984.

[15] H. Brézis and E. Lieb, "A relation between pointwise convergence of functions and convergence of functionals," Proceedings of the American Mathematical Society, vol. 88, no. 3, pp. 486490, 1983.

[16] M. Struwe, Variational Methods: Applications to Nonlinear Partial Differential Equations and Hamiltonian Systems, vol. 34 of Results in Mathematics and Related Areas (3), Springer, Berlin, Germany, 2nd edition, 1996.

[17] J. L. Vázquez, "A strong maximum principle for some quasilinear elliptic equations," Applied Mathematics and Optimization, vol. 12, no. 3, pp. 191-202, 1984. 


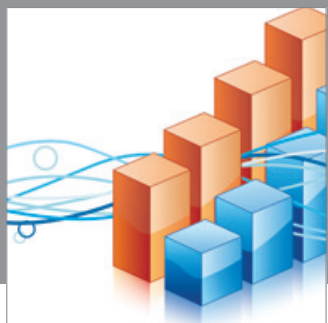

Advances in

Operations Research

mansans

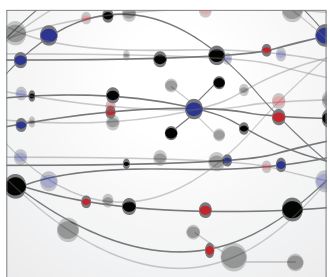

The Scientific World Journal
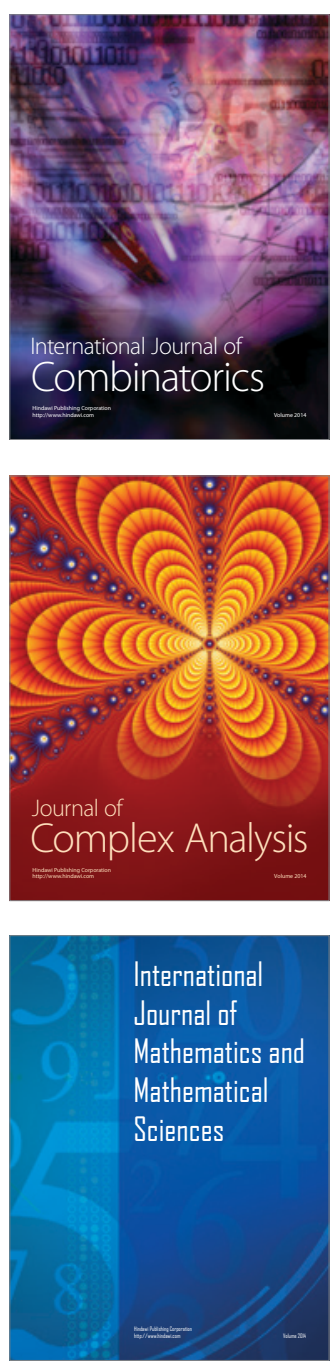
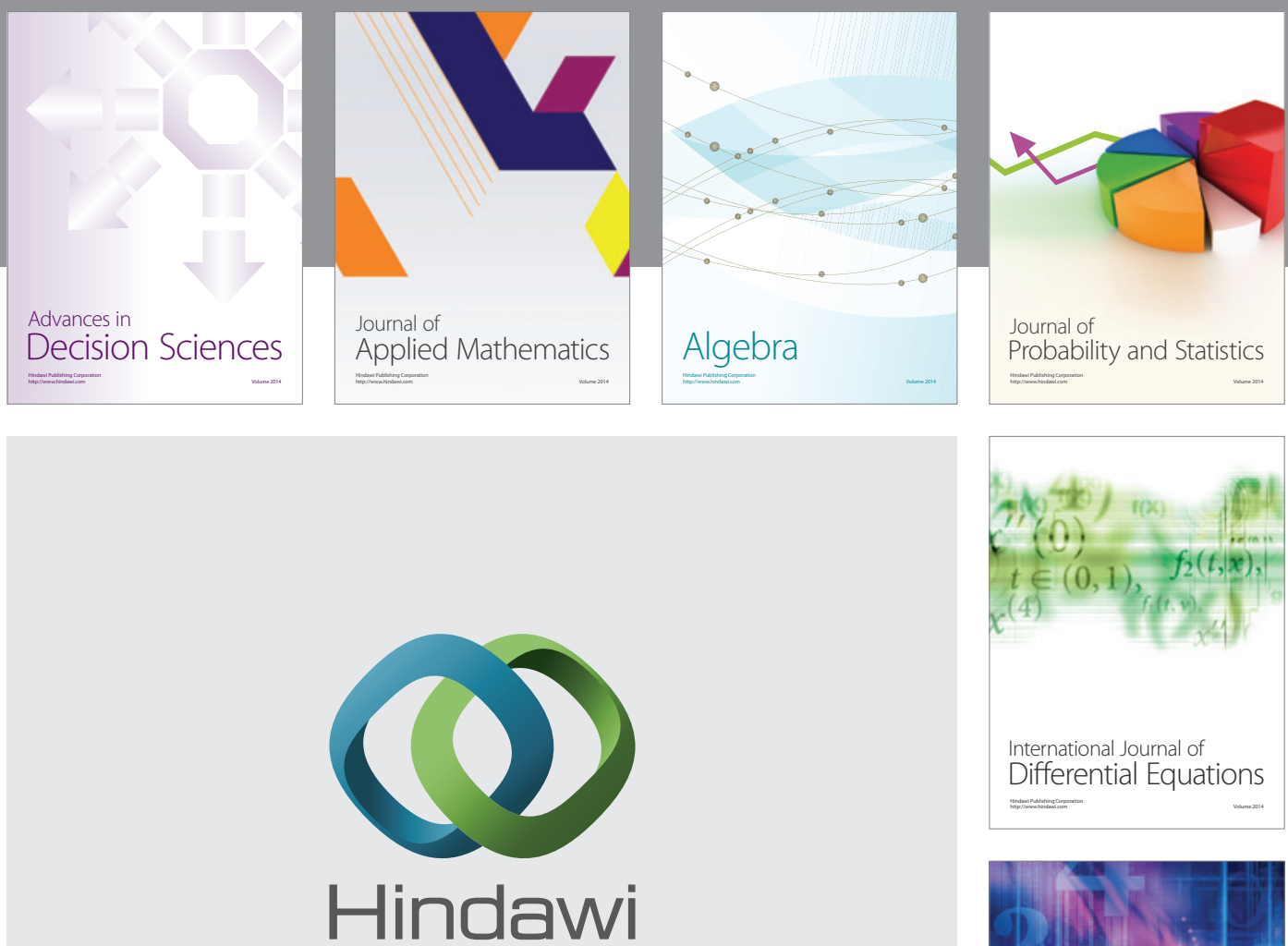

Submit your manuscripts at http://www.hindawi.com
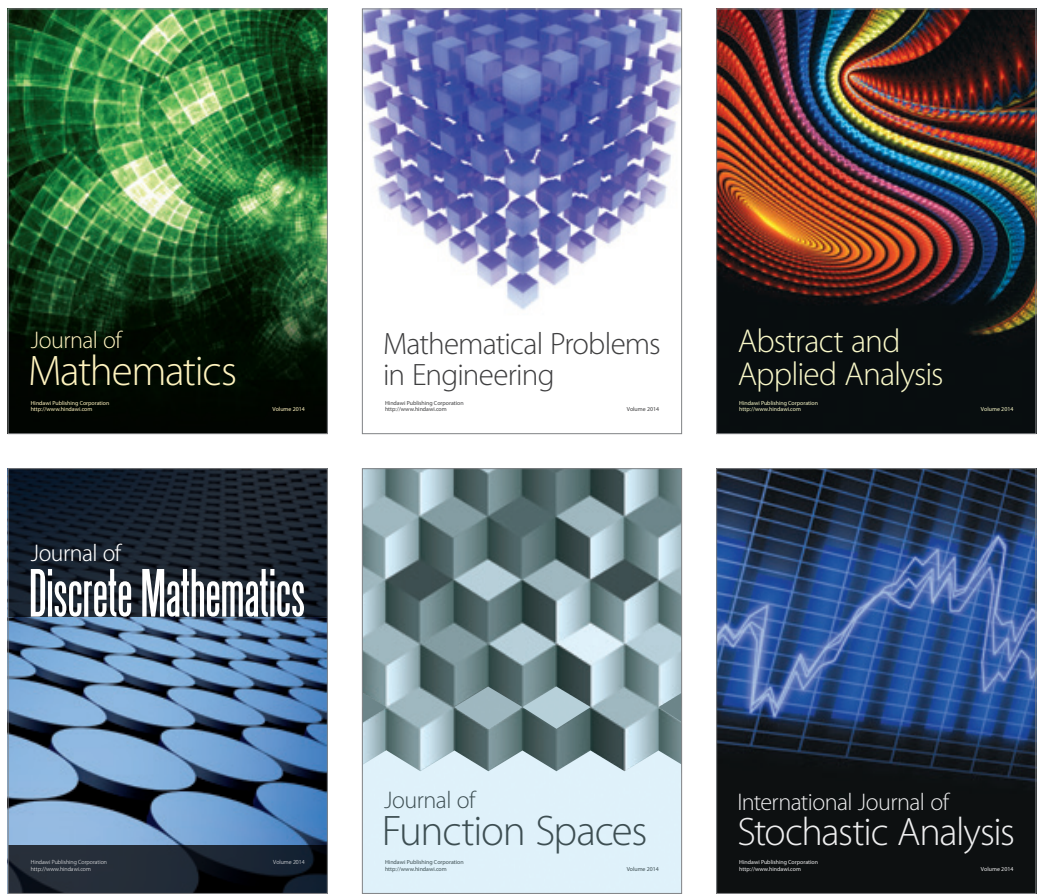

Journal of

Function Spaces

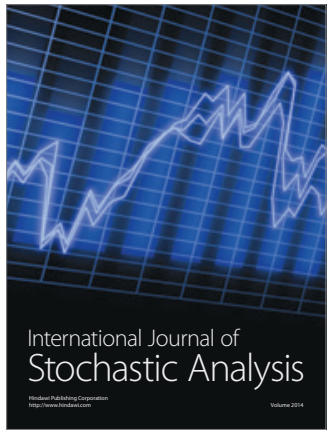

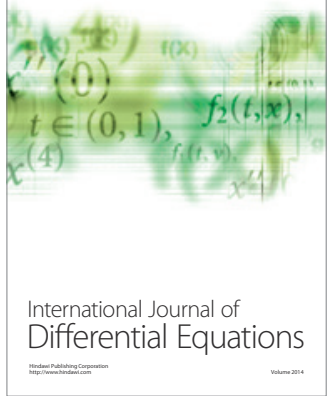
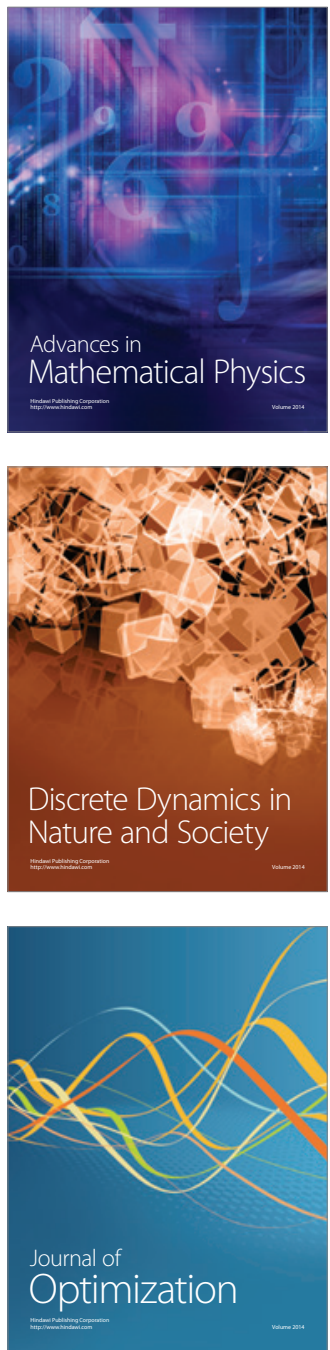